\title{
Propuesta de alineación del Sistema Integrado de Gestión del ICBF Regional Bogotá con las normas ISO 9001:2015, ISO 14001:2015 y OHSAS 18001:2007
}

Proposal for the alignment of the ICBF Bogotá Regional Integrated Management System with standards ISO 9001:2015, ISO 14001:2015 and OHSAS 18001:2007

Proposta de formação do Sistema Inteǵrado de Gestão do ICBF Regional Bogotá com as normas ISO 9001:2015, ISO 14001:2015 e OHSAS 18001:2007

Melco Javier Leuro Rodrígue****

Especialista en Administración y Gerencia de Sistemas de Calidad

* $\quad$ Artículo de resultado de investigación. DOI: http://dx.doi.org/10.15332/s2145-1389.2017.0002.03

* Magíster en Calidad y Gestión Integral. Especialista en Administración y Gerencia de Sistemas de la Calidad. Ingeniero Industrial. Profesional de apoyo en el Instituto Colombiano de Bienestar Familiar. Correo electrónico: melcojavier@yahoo.com. 


\section{RESUMEN}

Las instituciones públicas deben generar resultados eficaces y los Sistemas Integrados de Gestión se implementan como una herramienta administrativa que permite direccionarlas estratégicamente hacia sus objetivos. Sin embargo, en aquellas instituciones públicas que los han implementado, se presentan dificultades de tipo técnico, y peor aún, dificultades en el nivel laboral, al enfrentarse con situaciones controversiales en el ámbito del desarrollo de estos sistemas de gestión. De igual forma, la actualización de las normas técnicas internacionales ISO 9001 e ISO 14001 bajo la estructura de alto nivel, genera la necesidad de identificar las brechas existentes entre los sistemas implementados y los sistemas de gestión actualizados que sean herramientas reales, direccionadoras en la consecución de los objetivos estratégicos de la organización, herramientas que no discutan con la cultura organizacional impuesta y sean en cambio el agente dinamizador, que gestione la mejora al interior de la organización, aumentando la eficiencia, eficacia y ante todo efectividad visualizada en la sociedad. Este proyecto pretende dar las respuestas a las situaciones presentadas en una organización pública de Colombia, el Instituto Colombiano de Bienestar Familiar (ICBF) Regional Bogotá, institución donde se encuentra implementado un Sistema Integrado de Gestión bajo las pasadas versiones (NTC-ISO 9001:2008 y NTC-ISO 14001:2004) y SySO (NTC-OHSAS 18001:2007) omitiendo requisitos estipulados en las normas bajo las nuevas versiones, enfoques novedosos respecto de los beneficios que puede generar la implementación de un eficaz sistema de gestión que se desarrolle de forma integral al interior de la organización.

Palabras clave: estructura de alto nivel, organización pública y sistema integrado de gestión.

\section{ABSTRACT}

Public institutions must generate effective results and Integrated Management Systems (IMS) are implemented as an administrative tool that allows them to be strategically directed towards their objectives. However, in those public institutions that have implemented IMS, there are difficulties of a technical nature, and worse, difficulties in the labor market, when faced with controversial situations in the field of development of these management systems. In addition to, the updating of the international technical standards ISO 9001 and ISO 14001 under the high level structure generates the need to identify the existing gaps between the implemented systems and the updated management systems that are real tools, guiding in the attainment of the Strategic objectives of the organization, tools that do not discuss with the imposed organizational culture and instead are the dynamizing agent, that manages the improvement within the organization, increasing efficiency and above all effectiveness visualized in society. This project aims to provide answers to the situations presented in a public organization in Colombia, the Instituto Colombiano de Bienestar Familiar (ICBF) Regional Bogotá, institution where an Integrated Management System is implemented under the past versions (NTC- ISO 9001:2008 and NTC-ISO 14001:2004) and NTC-OHSAS 18001:2007 omitting requirements stipulated in the standards under new versions, novel approaches to the benefits that can be generated by the implementation of an effective management system That is developed in an integral way within the organization.

Keywords: high level structure, public organization and integrated management system. 


\section{RESUMO}

As instituições públicas devem gerar resultados efetivos e os Sistemas Integrados de Gestão são implementados como uma ferramenta administrativa que permite ser direcionada estrategicamente aos seus objetivos. No entanto, nas instituições públicas que implementaram o sistema, se apresentam dificuldades técnicas e pior ainda, dificuldades a nível laboral, quando confrontados com situações controversas no campo do desenvolvimento destes sistemas de gestão.

Da mesma forma, a atualização das normas técnicas internacionais ISO 9001 e ISO 14001 sob a estrutura de alto nível gera à necessidade de identificar as lacunas entre os sistemas implantados e os sistemas de gestão atualizados que sejam ferramentas reais, orientadoras no cumprimento dos objetivos estratégicos da organização, ferramentas que não discuta com a cultura organizacional imposta, que seja, por outro lado, o ator dinâmico, que gestione a melhoria interna da organização, aumentando a eficiência, eficácia e sobre tudo efetividade vista na sociedade. Este projeto visa fornecer respostas para às situações apresentadas em uma organização pública da Colômbia, o Instituto Colombiano de Bem Estar Familiar (ICBF) Regional Bogotá, instituição onde encontra-se implementado um Sistema Integrado de Gestão nas versões passadas (NTC-ISO 9001:2008 e NTG-ISO 14001:2004) e SySO (NTG-OHSAS 18001:2007) omitindo os requisitos estipulados nas normas sob às novas versões, abordagens inovadoras no que diz respeito aos benefícios que podem ser gerados pela implementação de um sistema eficaz de gestão desenvolvido de forma abrangente para 0 centro da organização.

Palavras-chave: estrutura de alto nível, organização pública e sistema integrado de gestão.

\section{INTRODUCCIÓN}

Las instituciones sin importar su condición (particular o pública), requieren ser competitivas en la sociedad actual. Casos como la eliminación del Departamento Administrativo de Seguridad (DAS), en Colombia, aparte de las razones políticas, evidencian la criticidad de la eficacia de las instituciones como factor para su continuidad. En este sentido, la sostenibilidad de las instituciones del Estado no se debe afianzar en su condición de monopolio por ser entidad pública sino en la obligación social con la que sus funcionarios presten el servicio a los usuarios.

Para conocer algunos aspectos de la organización tema del estudio, se enuncia su presentación según el Manual del Sistema Integrado de Gestión:

El Instituto Colombiano de Bienestar Familiar -ICBF- "Cecilia de la Fuente de Lleras", es un establecimiento público descentralizado, con personería jurídica, autonomía administrativa y patrimonio propio, perteneciente al Sector de la Inclusión Social y la Reconciliación, adscrito al Departamento Administrativo para la Prosperidad Social mediante Decreto 4156 del 3 de noviembre de 2011, creado por la Ley 75 de 1968, reorganizado conforme a lo dispuesto por la Ley 7 de 1979 y en el Decreto 1084 de 2015.

El ICBF tiene por objeto propender y fortalecer la integración y el desarrollo armónico de la familia, proteger a los niños, niñas y adolescentes y garantizarles sus derechos. Para ello, coordina la integración funcional de las Entidades públicas y privadas que conforman el Sistema Nacional de Bienestar Familiar - SNBF para que participen armónica y racionalmente de acuerdo a su competencia (ICBF, 2016, p. 8).

En cuanto a su organización, enuncia:

La estructura orgánica del ICBF se encuentra reglamentada por el Decreto 987 de 2012, geográ- 
ficamente cuenta con una Sede de la Dirección General, treinta y tres (33) Direcciones Regionales en el nivel departamental y doscientos nueve (209) centros zonales en el nivel municipal, con cobertura nacional en los 1.123 municipios para ejecutar los programas y servicios del Instituto a favor de la niñez, la familia y las comunidades (p. 8).

Los sistemas de gestión se convierten pues en herramientas administrativas para lograr los objetivos propuestos por las instituciones, sean públicas o privadas. Los sistemas de gestión son ampliamente conocidos e implementados a nivel mundial.

\section{La implementación y certificación de sistemas de calidad (ISO 9001), medioambiental (14001) y sa- lud y seguridad ocupacional (OHSAS 18001) han sido una actividad importante para muchas orga- nizaciones y se han convertido en un fenómeno generalizado en todo el mundo [1]. Según una encuesta realizada por la Organización Interna- cional de Normalización (ISO), los registros mun- diales de ISO 9001:2000 superaron la marca de 670.399, y el número de organizaciones registradas en ISO 14001:1996 se acercó a 90.569 a finales de 2004 [2] (Zeng, Shi y Lou, 2007, pp. 1760-1767).}

Datos que a finales del 2006 crecieron en la norma ISO 9001 a más de un millón de organizaciones y en la ISO 14001 a más de 129.000 (Karapetrovic, Casadesus y Heras, 2010).

Pero en la actualidad ya no es suficiente un solo sistema de gestión, según sean los objetivos y metas propuestas por la institución, se podría pensar en la implementación de más de un sistema de gestión al interior de la organización.

Motivados por el mercado o por requerimientos internos las empresas a nivel mundial se preocupan por integrar de manera eficaz los sistemas de gestión implementados, tanto es así que "algunos países han creado incluso sus propios modelos de integración de sistemas de gestión, como por ejemplo, el caso de Australia, Dinamarca o España. En julio de 2008, la ISO publicó el manual 'El uso integrado de las normas del sistema de gestión”' (p. 534).

Un factor estudiado en relación a la implementación de más de un sistema de gestión en la misma organización es la secuencia de implementación, según Karapetrovic y otros (2010), en investigación realizada en 176 empresas que como mínimo aplicaban ISO 9001 y 14001, concluyen que: "En la mayoría de los casos, la secuencia de implementación seguirá la publicación de estándares, es decir, primero se introducirá SGC basado en ISO 9001, seguido por un SGA conforme a ISO 14001. Posteriormente, otras funciones se normalizarían, por ejemplo SySO de acuerdo con OHSAS 18001" (p. 534).

Aun cuando se aprecia el valor agregado que genera la implementación de un Sistema Integrado de Gestión en una empresa y está validado que "los diferentes sistemas de gestión proporcionan un marco operativo y un procedimiento, al mismo tiempo que apoyan la mejora continua"( Kauppila, Härkönen, y Väyrynen, 2015), también es cierto que en la práctica "se ha comprobado que es difícil tratar con sistemas de gestión separados que abarquen la calidad, el medio ambiente y la salud y la seguridad en el trabajo y que garanticen su alineación con la estrategia de la organización [5]" (Zeng et al., 2007, p. 1760).

Colombia a 2015 ocupaba el segundo puesto en Latinoamérica con relación al número de empresas certificadas en ISO 9001 (12.324 organizaciones con esta certificación). Los primeros cinco sectores que incluyen a estas empresas son la construcción (1.490), otros servicios (1.233), educación (1.199), transporte almacenamiento y comunicación (1.107) y comercio al por mayor y al por menor; reparaciones de vehículos de motor, motocicletas y artículos personales y de uso doméstico (729) (Vera, 2016). 
En cuanto al número de organizaciones certificadas en el estándar ISO 14001, Colombia de igual forma ocupaba el segundo lugar en Suramérica al año 2015. En el año 2004 se contaba con 217 organizaciones con dicha certificación, creciendo periódicamente desde entonces con tres picos significativos: en el año 2008, con un crecimiento de un $64 \%$ con respecto al año anterior; para el 2010, creciendo un $81 \%$; y en el 2013 se incrementó en un $93 \%$ el número de organizaciones con certificado 14001. Para la vigencia 2015 las certificaciones decrecieron en un $18 \%$ con respecto al año 2014, único período con tendencia negativa (Prieto, 2015).

Con relación a la seguridad y salud ocupacional en Colombia, las empresas que desean certificar sus sistemas SySO han aumentado considerablemente en los últimos años. Según estadísticas del Consejo Colombiano de Seguridad (CCS) (Portafolio, 15 de mayo de 2013), al año 2008, 31 empresas se inscribieron para certificarse en dicho sistema de gestión; a 2009, 52 hicieron lo propio; en 2010, 56 empresas se inscribieron en el CCS y en 2011 este numero creció, llegando en la vigencia 2012 a un total de 140 instituciones que deseaban certificar su sistema SySO con dicha entidad.

En el ICBF Regional Bogotá, una de las 33 regionales enunciadas anteriormente y que tiene asignados 17 centros zonales, se implementa un Sistema de Gestión Integral cuya historia comienza con el Sistema de Gestión de la Calidad desde el año 2004, en dos procesos específicos: Proceso Administrativo de Protección (PAP) y Adopciones; siendo esta regional y estos procesos los pilotajes para posteriormente ampliar el alcance de dicho sistema. Al 2008 el ICBF ha permeado el Sistema de Gestión de la Calidad a la totalidad de los procesos y propone la implementación del Sistema de Gestión Ambiental. Mas adelante se toma la decisión estratégica de implementar el Sistema de Gestión de Seguridad y Salud Ocupacional (SySO); siendo estos los sistemas de gestión que entran a conformar el
SIGE (Sistema Integrado de Gestión), como es conocido al interior de dicha institución.

Es de anotar, la implementación del Sistema de Gestión de Seguridad de la Información; sistema que fue certificado en una primera instancia, pero que posteriormente perdió el aval del ente certificador, razón por la cual en este momento su alcance se redujo a la Dirección de Información y Tecnología, dependencia de la Sede de la Dirección General, y que se encuentra en implementación para el resto de dependencias, regionales y procesos, razón por la cual no se tuvo en cuenta para el presente trabajo.

Un factor relevante en este tema se relaciona con las normas ISO 9001 y 14001, estándares internacionales que se actualizaron en septiembre de 2015, y se encuentra en desarrollo el diseño de la norma OHSAS 18001, como norma internacional (ISO 45001), modificaciones que deben ser tenidas en cuenta para el mantenimiento y actualización de estos tres sistemas de gestión dentro de la organización.

Con el ánimo de aumentar la alineación de las normas internacionales para el desarrollo de los sistemas de gestión, la Organización Internacional de Normalización (ISO) ha creado la estructura de alto nivel o Anexo SL, mediante el cual se estandariza la estructura de las normas de los sistemas de gestión; al respecto, Kauppila, Härkonen y Väyrynen (2015) comentan:

ISO 9001, ISO 14001 y OHSAS 18001 están experimentando actualmente revisiones significativas y sus versiones revisadas van a seguir la denominada estructura del Anexo SL desarrollada por ISO. Es un marco para proporcionar idéntica estructura, texto y términos y definiciones comunes para los estándares de sistemas de gestión (MSS). La estructura de alto nivel del Anexo SL de ISO proporciona una reforma significativa mediante la combinación de estructuras (Hotti, 2014, Pojasek, 2013). Algunos estándares 
ya están armonizados con el Anexo SL, y todos los ISO pretenden usar la estructura en todos los MSS nuevos y revisados (Warris y Tangen, 2012). Según Pojasek (2013), los cambios más significativos para los usuarios son los requisitos para definir la organización y el contexto interno y externo relacionado con un MS, identificar los procesos requeridos para el MS y abordar el riesgo y sus efectos (p. 232).

Ya que el ICBF Regional Bogotá implementó y mantiene un Sistema Integrado de Gestión, eje central de la investigación, es pertinente definir el concepto. Tejada y Peña (2009) lo definen como "determinación y adopción precisa de las partes (componentes) que conforman la organización como un todo y que se articulan alrededor de un propósito común" (p. 88).

Pero, ¿y para qué implementar sistemas integrados de gestión? Según estudio realizado por Karapetrovic, Casadesus y Heras (2010), las razones para implementar sistemas de gestión adicionales al interior de la empresa fueron en orden de importancia: la mejora de la imagen y el impacto social de la empresa, mejora en la eficiencia y el control de la organización, provisión de ventaja competitiva y reducción de los problemas y accidentes.

Estos diferentes factores generan diferencias entre el deber ser del Sistema Integrado de Gestión y el sistema implementado en la actualidad, brecha que si se lograse reducir, le implicaría al ICBF Regional Bogotá minimizar los reprocesos, utilizar con mayor eficiencia sus recursos, llegando así a aumentar la satisfacción de sus clientes, que para este caso son los niños, niñas y familias del distrito capital; aumentar el control de sus aspectos ambientales, beneficiando a la sociedad en general y disminuir los incidentes y accidentes, impactando principalmente a sus clientes internos y colaboradores.

En este contexto, se decantan tres preguntas rectoras desarrolladas en la investigación: ¿Cuál es la percepción del nivel de articulación de los Sistemas de Gestión en el ICBF Regional Bogotá? ¿Cuáles son los elementos de los estándares ISO 9001:2015 e ISO 14001:2015 que es necesario implementar en el Sistema Integrado de Gestión del ICBF Regional Bogotá?, y: ¿Cómo alinear el Sistema Integrado de Gestión del ICBF Regional Bogotá con las versiones 2015 de las normas ISO 9001 e ISO 14001?

Reconocer la percepción de aquellos que colaboran en el mantenimiento del Sistema Integrado de Gestión permite visibilizar las fortalezas y debilidades que se deben abordar para eliminar la brecha existente.

De igual forma, identificar los requisitos establecidos en las normas actualizadas y compararlos con la realidad institucional, se convierte en paso obligatorio para generar una propuesta que mitigue las diferencias evidenciadas.

Karapetrovic (2003), propone un proceso de siete pasos para lograr este objetivo: comienza especificando los objetivos de integración y determinando la factibilidad y alcance de un Sistema Integrado de Gestión, el segundo paso es elegir un modelo de integración que sirva como base de un SIG, el tercer paso decidir qué funciones (por ejemplo: calidad, medio ambiente, responsabilidad social) se deben incluir en el nuevo e integrado sistema, así como qué normas se utilizarán, a continuación estos requisitos deben ser integrados para proporcionar un conjunto de criterios de referencia a los que se les puede medir el SIG, posteriormente deberá realizarse un análisis de las carencias con respecto a los criterios integrados, esto es seguido por la alineación e integración de la documentación SIG, así como de objetivos, procesos y recursos específicos de la función. El último paso es mejorar.

Es necesario tener en cuenta, según Karapetrovic, que para experimentar los beneficios de un SIG, es deseable al menos alinear metas así como recursos y 
procesos importantes. "Si bien es bastante obvia la necesidad de alinear los objetivos específicos de la función, la armonización de los recursos, aunque no estén totalmente integrados, mejorará la cooperación y la comunicación entre sistemas, además de proporcionar ahorros significativos al minimizar los usos múltiples en todas las funciones" (p. 12).

\section{METODOLOGÍA}

La presente investigación tiene un enfoque mixto ya que se utiliza un conjunto de procesos de recolección de información, análisis y vinculación de datos cuantitativos y cualitativos en un mismo estudio respondiendo al objetivo principal del mismo (Hernández, Fernández y Baptista, 2014).

Teniendo en cuenta la aplicación, como instrumentos de investigación, de una encuesta y una matriz de identificación de requisitos según las tres normas de los Sistemas de Gestión de la Calidad, Ambiental y de Seguridad y Salud Ocupacional; se recaban datos cuantitativos y cualitativos.

Se recurre a esta metodología por la complejidad del tema tratado; sería impreciso decir que la brecha entre el Sistema Integrado de Gestión implementado en el ICBF Regional Bogotá, se puede disminuir en relación a las nuevas versiones de las normas internacionales con tan solo la verificación de los requisitos estipulados en las versiones basada en la estructura de alto nivel, al igual que sería un error inferir que solo se debería acudir a la percepción que la institución posee del sistema para cerrar la brecha existente, tema de la presente investigación.

Investigación concurrente. Los instrumentos adoptados para la realización de la investigación, fundamentalmente la encuesta y la matriz, se desarrollan paralelamente y ninguna es insumo de la otra; aun cuando para alcanzar el objetivo de la investigación, el plan de trabajo final se basa en el análisis de las dos fuentes.

\section{Diseño anidado o incrustado concurrente de modelo domi-} nante. De acuerdo con los apartes anteriores, la presente investigación se enmarca en un diseño mixto, de tipo concurrente, clasificándose específicamente en un "Diseño anidado o incrustado concurrente de modelo dominante", ya que, como se enunció en el anterior aparte, aun cuando sería un error garantizar que se obtendrían resultados con una sola fuente de información, es justo decir, que el análisis de los requisitos de la norma direcciona los resultados de la investigación, ya que son estos los que se deberán implementar, con ayuda y respaldo de la cultura organizacional, evidenciada a través de la entrevista de percepción.

Encuesta de percepción. Se realiza encuesta de percepción estructurada en tres categorías: a) concepción general - posición del servidor público/contratista en relación al Sistema Integrado de Gestión, b) ítems transversales a los tres sistemas de gestión y, c) percepción de las herramientas de mejora. En total la encuesta desarrolla 18 preguntas (3 para la primera categoría, 8 para la segunda y 7 para la tercera) con cinco posibilidades de respuesta (totalmente en desacuerdo, en desacuerdo, de acuerdo, totalmente de acuerdo y desconozco sobre el tema preguntado).

La validación del instrumento se realiza con cuatro profesionales. Dos expertos técnicos, ingenieros industriales, una especialista en Gestión de la Calidad y el otro especialista en Aseguramiento de la Calidad; los dos con vasta experiencia en la implementación y mantenimiento de sistemas de gestión. De igual forma realizan aportes dos profesionales que laboran como "Referentes de Calidad" en el ICBF Regional Bogotá, funcionarios que tienen como función el mantenimiento y mejora del Sistema Integrado de Gestión al interior de la regional, pares del autor del presente trabajo. 
Con el objetivo de dinamizar la implementación (en un comienzo) y el mantenimiento y mejora del sistema integrado de gestión (hoy día), en la institución se creó una figura llamada "GAMI" (Grupo de Apoyo para el Mejoramiento Organizacional); a través del tiempo esta figura evolucionó en el "Promotor ÉPICO", servidores públicos o contratistas que independientemente de su rol o funciones, apoyan al coordinador de su dependencia (grupo o centro zonal) en la gestión de las herramientas específicas solicitadas por los sistemas de gestión como son: tablero de control, seguimiento y control de las acciones de mejora, mapas de riesgo, seguimiento a compromisos de revisión por la Dirección, en actividades como sensibilizaciones y socializaciones, etc.

Estos promotores ÉPICO (sigla de Estrategia Permanente de Innovación y Cambio Organizacional, estrategia implementada en el ICBF), han tomado tanta fuerza y relevancia para el mantenimiento del Sistema Integrado de Gestión, hasta el punto de que en actos administrativos y procedimientos internos ya son tenidos en cuenta como puntos clave del desarrollo de los procesos. Tanto es así, que la Resolución 10232 del 30 de noviembre de 2015, por la cual se reorganiza el Sistema Integrado de Gestión y se dictan otras disposiciones, les ha fijado funciones.

Una vez validado el instrumento, se aplica mediante plataforma virtual (Google Forms) direccionándolo a la población objetivo, los promotores ÉPICO del $100 \%$ de las dependencias de la Regional Bogotá, obteniéndose respuesta de la totalidad de la población seleccionada.

El manejo de la información se realiza mediante "libro Excel", consolidando frecuencias de respuestas y generando valores relativos tanto por categorías como por pregunta realizada; dichos datos se consolidan por tipología de nivel organizacional (Regional y Centro Zonal).

Matriz de normas implementadas. El segundo instrumento utilizado en la presente investigación, es la matriz de normas implementadas. Se plasman en libro Excel los "debes" de las normas de calidad, ambiental y seguridad y salud ocupacional, alineando por filas cada requisito establecido.

Una vez se refleja la totalidad de los requisitos, se procede a analizar el cumplimiento de cada uno de estos al interior del ICBF Regional Bogotá, obteniendo tres clasificaciones de requisitos: cumple, no cumple, falta. Para la primera clasificación (cumple), se obtienen los requisitos de las normas que se ejecutan en el sistema de gestión implementado en la organización y en los cuales, consecuentemente, existe evidencia, la cual se plasma en la herramienta. Para la segunda clasificación, (no cumple), se obtienen los requisitos que la organización no ha implementado en sus sistemas de gestión. Por ultimo, la tercera clasificación (falta), corresponde a los requisitos que, aunque tienen algún tipo de avance, no están implementados en su totalidad. La brecha existente radica en las dos últimas tipologías de clasificación realizadas.

En la herramienta se plasma para cada uno de los requisitos clasificados en no cumple o falta, la propuesta con la cual se podría eliminar la brecha evidenciada.

La institución apoyó la recolección de datos hasta el punto de que en la realización de la encuesta de percepción, quedó como compromiso de revisión por la Dirección, corte a 30 de junio de 2016.

De igual forma, para el análisis de cumplimiento de los requisitos de la norma, el autor adicional a la información primaria se influenció en dos ejercicios institucionales realizados durante el desarrollo del presente trabajo. El primero se dio en un espacio de capacitación, actualización de la norma ISO 9001:2015, espacio en el que participaron "Referentes de Calidad" y coordinadores de grupos de Planeación y Sistemas de varias regionales del ICBF. Espacio en el cual se realizó ejercicio de comparación de los requisitos de las normas vs. 
cumplimiento por parte de la organización. El segundo ejercicio institucional que influyó en los resultados del análisis de cumplimiento e identificación de brecha existente, fue el desarrollo de la "Lista de chequeo diagnóstico POSIGE", ejercicio que consistió en la aplicación de una lista de chequeo al interior de cada centro zonal y sede regional en todo el país (por supuesto, incluyendo la sede Regional Bogotá y sus 17 centros zonales) con el objetivo de generar un diagnóstico que sirviera como insumo en el desarrollo de un plan de transición a las nuevas versiones de las normas internacionales.

\section{RESULTADOS Y DISCUSIÓN}

En cuanto a los resultados de la investigación, se obtiene lo siguiente: La encuesta de percepción indica que en el nivel regional (R) se percibe que el SGC ayuda a satisfacer las necesidades del usuario, por lo que sería un sistema eficaz; en el nivel zonal (Z) tan solo un poco mas de la decima parte de la población encuestada está totalmente de acuerdo con que el SGC aporta a la satisfacción del usuario, aun cuando se logra equilibrio entre los dos niveles (R: $78 \%$; Z: $77 \%$ ) en obtener una posición favorable al logro de la razón de ser del SGC. Para el SGA, se obtiene que en el nivel regional existe una percepción de casi el 90 \% de aceptación en los logros de control y mitigación de impactos ambientales, pero la tercera parte del nivel zonal no está de acuerdo con este logro. En la sede regional, todos los encuestados perciben eficacia en el desarrollo del SySO, mientras que en el nivel zonal aquellos que están de acuerdo y totalmente de acuerdo con la proposición llegan a casi un $90 \%$. Es interesante visualizar esta percepción teniendo en cuenta que en la vigencia 2016 se han reportado un mayor numero de accidentes laborales que en la vigencia 2015.

A nivel regional, casi cuatro quintas partes de los grupos no están de acuerdo de que exista liderazgo por parte de las coordinaciones respectivas y el $89 \%$ de los encuestados a nivel zonal está de acuerdo o totalmente de acuerdo con este liderazgo. El factor compromiso, se percibe casi en un $50 \%$ de la población y en el otro $50 \%$ se observa la falta de compromiso por parte del personal en relación a los sistemas de gestión.

A nivel regional, casi el $90 \%$ y en el nivel zonal casi el $80 \%$ de los encuestados, se encuentran de acuerdo 0 totalmente de acuerdo con la efectividad de la comunicación que se realiza en relación al Sistema Integrado de Gestión.

Con el asunto de recursos, tema sensible en una entidad pública, se obtiene que en el nivel regional el $11 \%$ y en el nivel zonal el $24 \%$ de los encuestados, está totalmente en desacuerdo con que exista la asignación de recursos necesarios para la implementación y mantenimiento de los sistemas de gestión. A estos resultados extremos, les siguen unos porcentajes de 67 y $53 \%$ de población (nivel regional y zonal, respectivamente) que no considera que exista la asignación de los recursos requeridos, lo que implica que más de la mitad de los encuestados no visualiza los recursos requeridos para lograr una verdadera implementación o mantenimiento real de los sistemas de gestión.

Casi el $90 \%$ de los encuestados en el nivel regional considera que existe coherencia entre las políticas de los sistemas de gestión y los objetivos estratégicos de la institución. Lo propio sucede en el nivel zonal.

El 47 \% de la población (casi la mitad) esta en desacuerdo con que los servidores públicos y contratistas del ICBF Regional Bogotá, sean conscientes de los requisitos legales aplicables y otros requisitos existentes en el ICBF.

Más de la quinta parte de la población de la regional (22\%) desconoce sobre la identificación de las partes interesadas del sistema. Coherentemente, el resulta- 
do a nivel regional arroja un $22 \%$ de desconocimiento frente a las necesidades de dichas partes interesadas. El $56 \%$, en esta ocasión, considera que no se han identificado las necesidades y expectativas de las partes interesadas, tan solo un $22 \%$ estaría de acuerdo con la identificación de estos requerimientos. En el nivel zonal, se presenta un $6 \%$ de desconocimiento frente al tema y un $53 \%$ está en desacuerdo con dicha identificación.

El $100 \%$ de la población a nivel regional está, o de acuerdo (78\%) o totalmente de acuerdo (22\%), con la mejora de los sistemas de gestión gracias a la aplicación de auditorías internas. En el nivel zonal, el $59 \%$ está de acuerdo y el $35 \%$ esta totalmente de acuerdo con esta premisa.

Dos terceras partes del nivel regional (67\%) consideran que el seguimiento a los indicadores aporta a la mejora de los sistemas de gestión, mientras que en el nivel zonal cuatro quintas partes de la población (83\%) lo considera así.

Un $44 \%$ de la población encuestada en el nivel regional está de acuerdo con el aporte de los servicios no conformes (SNC); en contraposición, a nivel zonal un $82 \%$ de los encuestados considera que dicha herramienta sí aporta a la mejora del SGC.

Alrededor de la quinta parte de la población encuestada no está de acuerdo con el aporte de la revisión por la Dirección a la mejora de los sistemas (R: 22\%; Z: 18 $\%)$. De igual forma, aproximadamente la tercera parte de la población encuestada no está de acuerdo con el aporte de la administración del riesgo para la mejora de los sistemas de gestión.

En los dos niveles se identifica una percepción positiva cercana a las cuatro quintas partes de la población encuestada (cerca del $80 \%$ ) en relación al aporte de las acciones correctivas a la mejora del sistema de gestión.
La percepción de la articulación de los sistemas es divergente en los dos niveles aplicados. En el nivel regional el $56 \%$ de la población considera que los sistemas de gestión están articulados con un $11 \%$ en total desacuerdo y un $33 \%$ en desacuerdo; lo que plantea un panorama, dividido casi a la mitad en la percepción de este enunciado. En el nivel zonal se obtiene un $59 \%$ de respuestas en desacuerdo $(6 \%$ en total desacuerdo), y cerca de la cuarta parte de acuerdo con la articulación de los sistemas y una quinta parte con un total acuerdo de articulación; obteniéndose de nuevo una participación dividida en la percepción frente a este punto.

En cuanto a la matriz de normas implementadas, se obtienen los siguientes resultados: los debes de la norma ISO 9001:2015, se cumplen en un $90 \%$, le falta, el $10 \%$ de los requisitos y no cumple, un $0,3 \%$ de los requisitos de esta norma.

La ISO 14001:2015 se cumple en un $84 \%$, le falta, un $15 \%$ y definitivamente no cumple, un $1,1 \%$ de los "debes" de la nueva versión. La OHSAS 18001:2007 cumple en el $91 \%$ de sus requisitos, le falta, un $8 \%$, y no cumple, en un $1 \%$

Para dar lectura a los resultados del cumplimiento de los requisitos de las tres normas en un sistema integrado de gestión, se definen 7 zonas posibles en donde se pueden ubicar dichos requisitos (ver Figura 1).

$\mathrm{El}$ área $\mathrm{A}$, corresponde a los requisitos establecidos y articulados en las tres normas, el área B corresponde a los requisitos articulados entre la 9001 y la 14001; el área $\mathrm{C}$, a los requisitos articulados entre la 9001 y la 18001; el área D, a los "debes" alineados entre la 14001 y la 18001; en las zonas E, F y G, se ubican aquellos requisitos que no están alineados con ninguna otra norma, son exclusivos para cada sistema de gestión. 


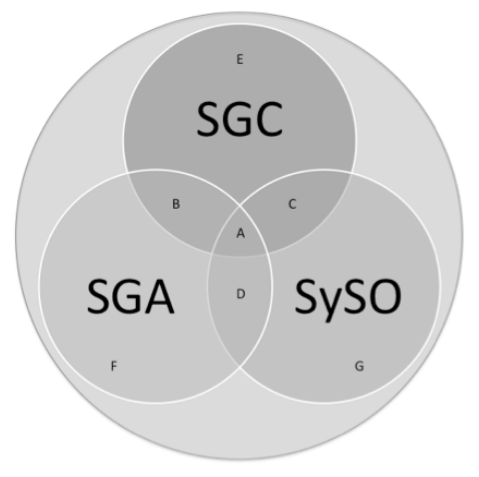

Figura 1. Áreas de articulación del Sistema Integrado de Gestión en el ICBF Regional Bogotá.

Fuente: el autor.

En las siguientes tablas se visualiza el grado de cumplimiento en cada una de estas zonas:

Tabla 1. Zona A

\begin{tabular}{|l|c|c|c|c|c|c|c|c|}
\cline { 2 - 9 } \multicolumn{1}{c|}{} & $\begin{array}{c}\text { NTC-ISO 9001:2015 } \\
\text { NTC-ISO 14001:2015 } \\
\text { NTC-OHSAS 18001:2007 }\end{array}$ & $\%$ & $\begin{array}{c}\text { NTC-ISO } \\
\mathbf{9 0 0 1 : 2 0 1 5}\end{array}$ & $\%$ & $\begin{array}{c}\text { NTC-ISO } \\
\mathbf{1 4 0 0 1 : 2 0 1 5}\end{array}$ & $\%$ & $\begin{array}{c}\text { NTC-OHSAS } \\
\mathbf{1 8 0 0 1 : 2 0 0 7}\end{array}$ & $\%$ \\
\hline Intersección & 61 & & & & & & & \\
\hline Cumple & 46 & $75 \%$ & 56 & $92 \%$ & 48 & $79 \%$ & 55 & $90 \%$ \\
\hline No cumple & 0 & $0 \%$ & 0 & $0 \%$ & 0 & $0 \%$ & 0 & $0 \%$ \\
\hline Falta & 4 & $7 \%$ & 5 & $8 \%$ & 13 & $21 \%$ & 6 & $10 \%$ \\
\hline SUMATORIA & $\mathbf{5 0}$ & $\mathbf{8 2} \%$ & $\mathbf{6 1}$ & $\mathbf{1 0 0} \%$ & $\mathbf{6 1}$ & $\mathbf{1 0 0 \%}$ & $\mathbf{6 1}$ & $\mathbf{1 0 0 \%}$ \\
\hline
\end{tabular}

Fuente: el autor.

Tabla 2. Zona B.

\begin{tabular}{|l|c|c|c|c|c|c|}
\cline { 2 - 7 } & $\begin{array}{c}\text { NTC-ISO 9001:2015 } \\
\text { NTC-ISO 14001:2015 }\end{array}$ & $\%$ & $\begin{array}{c}\text { NTC-ISO } \\
\mathbf{9 0 0 1 : 2 0 1 5}\end{array}$ & $\%$ & $\begin{array}{c}\text { NTC-ISO } \\
\mathbf{1 4 0 0 1 : 2 0 1 5}\end{array}$ & $\%$ \\
\hline Intersección & 56 & & & & & \\
\hline Cumple & 47 & $84 \%$ & 54 & $96 \%$ & 48 & $86 \%$ \\
\hline No cumple & 0 & $0 \%$ & 0 & $0 \%$ & 1 & $2 \%$ \\
\hline Falta & 1 & $2 \%$ & 2 & $4 \%$ & 7 & $13 \%$ \\
\hline SUMATORIA & $\mathbf{4 8}$ & $\mathbf{8 6 \%}$ & $\mathbf{5 6}$ & $\mathbf{1 0 0} \%$ & $\mathbf{5 6}$ & $\mathbf{1 0 0 \%}$ \\
\hline
\end{tabular}


Tabla 3. Zona C.

\begin{tabular}{|l|c|c|c|c|c|c|}
\cline { 2 - 7 } \multicolumn{1}{c|}{} & $\begin{array}{c}\text { NTC-ISO 9001:2015 } \\
\text { NTC-OHSAS 18001:2007 }\end{array}$ & $\%$ & $\begin{array}{c}\text { NTC-ISO } \\
\mathbf{9 0 0 1 : 2 0 1 5}\end{array}$ & $\begin{array}{c}\text { NTC-OHSAS } \\
\mathbf{1 8 0 0 1 : 2 0 0 7}\end{array}$ & $\%$ \\
\hline Intersección & 5 & & & & & \\
\hline Cumple & 5 & $100 \%$ & 5 & $100 \%$ & 5 & $100 \%$ \\
\hline No cumple & 0 & $0 \%$ & 0 & $0 \%$ & 0 & $0 \%$ \\
\hline Falta & 0 & $\mathbf{1 0 0} \%$ & $\mathbf{5}$ & $\mathbf{1 0 0 \%}$ & $\mathbf{5}$ & $\mathbf{1 0 0 \%}$ \\
\hline SUMATORIA & $\mathbf{5}$ & & & & 0 & $0 \%$ \\
\hline
\end{tabular}

Fuente: el autor.

Tabla 4. Zona D.

\begin{tabular}{|l|c|c|c|c|c|c|}
\cline { 2 - 7 } \multicolumn{1}{c|}{} & $\begin{array}{c}\text { NTC-ISO 14001:2015 } \\
\text { NTC-OHSAS 18001:2007 }\end{array}$ & $\%$ & $\begin{array}{c}\text { NTC-ISO } \\
\mathbf{1 4 0 0 1 : 2 0 1 5}\end{array}$ & $\begin{array}{c}\text { NTC-OHSAS } \\
\mathbf{1 8 0 0 1 : 2 0 0 7}\end{array}$ & $\%$ \\
\hline Intersección & 20 & & & & & \\
\hline Cumple & 16 & $80 \%$ & 18 & $90 \%$ & 17 & $85 \%$ \\
\hline No cumple & 0 & $0 \%$ & 0 & $0 \%$ & 0 & $0 \%$ \\
\hline Falta & 1 & $5 \%$ & 2 & $10 \%$ & 3 & $15 \%$ \\
\hline SUMATORIA & $\mathbf{1 7}$ & $\mathbf{8 5 \%}$ & $\mathbf{2 0}$ & $\mathbf{1 0 0 \%}$ & $\mathbf{2 0}$ & $\mathbf{1 0 0 \%}$ \\
\hline
\end{tabular}

Fuente: el autor.

Tabla 5. Zonas E, F y G.

\begin{tabular}{|l|c|c|c|}
\cline { 2 - 4 } & NTC-ISO 9001:2015 & NTC-ISO 14001:2015 & NTC-OHSAS 18001:2007 \\
\hline Intersección & 183 & 41 & 65 \\
\hline Cumple & 159 & 36 & 60 \\
\hline No cumple & 1 & 1 & 2 \\
\hline Falta & 23 & 4 & 3 \\
\hline SUMATORIA & $\mathbf{1 8 3}$ & $\mathbf{4 1}$ & $\mathbf{6 5}$ \\
\hline
\end{tabular}

Fuente: al autor 
En cuanto a la discusión de los citados resultados, se infiere: los resultados obtenidos según encuesta de percepción son coherentes con el contexto del ICBF Regional Bogotá; las percepciones del cumplimiento en los objetivos de los sistemas de gestión, están directamente relacionadas con el nivel de madurez que tiene el SIG según el tiempo de establecimiento. Las falencias sentidas en el liderazgo y compromiso por parte de los responsables de proceso y de los funcionarios se dan por la cultura y contraposición a ser medidos o a ser evaluados; aún falta concientizar el trabajo basado en resultados. No se aguardaba la conformidad existente con los canales de comunicación empleados en la institución, fortaleza que no se esperaba evidenciar. En cuanto a los recursos, definitivamente era una apuesta casi segura, que se presentara inconformismo en relación a la cantidad de recursos otorgados para el mantenimiento del sistema. Una fortaleza sentida en la institución es el direccionamiento estratégico, fortaleza reflejada en la relación existente entre los objetivos del sistema y las metas institucionales. Como debilidad, se evidencia tanto el desconocimiento de los requisitos legales aplicables como de las partes interesadas y sus necesidades, factor relevante, más aún con las nuevas versiones de las normas internacionales.

En cuanto al punto de mejora, se resalta el posicionamiento que tienen las auditorías internas como herramienta de mejora. Aun cuando la mayoría percibe a los indicadores de gestión como herramientas que propenden por el aumento en la capacidad del sistema para el cumplimiento de requisitos, no se puede invisibilizar la tercera parte de la población que no lo considera así. De igual forma, ya el sistema interiorizó a las no conformidades como factores que influyen en la mejora de los procesos y no como llamados de atención personales, percepción que se vivía tiempo atrás. Aun cuando un alto porcentaje está de acuerdo con el aporte realizado por la administración de riesgos, revisión por la Dirección y acciones correctivas, aun queda entre un 20 y un $30 \%$ de la población que no lo percibe de esa forma.

El resultado que consolida la situación actual de la institución en relación con el problema inicial de la investigación, permite inferir que el trabajo es totalmente vigente con las necesidades de la organización, casi un $50 \%$ de la población considera que falta articulación entre los sistemas que conforman el Sistema Integrado de Gestión en el ICBF Regional Bogotá.

En relación con los resultados arrojados por la matriz de normas implementadas, es positivamente sorprende que en general, la brecha existente es de tan solo un $12 \%$. Aun cuando es necesario implementar acciones, estas no representarán un esfuerzo mayúsculo en comparación con las actividades de mantenimiento del sistema.

\section{CONCLUSIONES}

Se identifica la percepción de la articulación existente entre los Sistemas de Gestión de la Calidad, Ambiental y de Seguridad y Salud en el ICBF Regional Bogotá, mediante la muestra del $100 \%$ de los promotores ÉPICO de los centros zonales y grupos de la regional, logrando información de la visualización existente por parte de los servidores públicos y contratistas del Sistema Integrado de Gestión, sus puntos transversales y las herramientas de mejora existentes.

Información mediante la cual se puede asegurar que el Sistema Integrado de Gestión es visto como herramienta que permite lograr los resultados previstos en cada uno de los sistemas de gestión, que es necesario aumentar el liderazgo a nivel regional, y que en el nivel zonal este principio está debidamente vivenciado; que es necesario fortalecer el compromiso hacia el logro de objetivos y conocimiento de los requisitos lega- 
les por parte de los funcionarios; que sorpresivamente se percibe a los canales de comunicación existentes como suficientes y eficaces para transmitir la información pertinente al Sistema Integrado de Gestión; que de acuerdo con la experiencia se evidencia desacuerdo con la asignación de recursos para el cumplimiento de requisitos; como fortaleza se percibe coherencia entre las políticas y objetivos institucionales; como debilidad y aspecto a mejorar, no se tienen en cuenta todas las partes interesadas ni sus necesidades para planificar ni implementar el Sistema Integrado de Gestión; que de forma genérica se percibe a las acciones de mejora como tales y que sustentados en la razón de ser de este trabajo de investigación, se advierte desarticulación entre los sistemas de gestión implementados. Información por la cual se puede asegurar que se cumplió con el primer objetivo específico del trabajo.

Se identifican los elementos necesarios a implementar en los Sistemas de Gestión de la Calidad, Ambiental y de Seguridad y Salud en el ICBF Regional Bogotá, mediante la comparación de los requisitos exigidos por las normas en relación con la evidencia que demuestra su aplicación o no al interior de esta regional.

Esta comparación arroja un resultado del $12 \%$ de requisitos por implementar para lograr la alineación del Sistema Integrado de Gestión con las normas en sus últimas versiones según la estructura de alto nivel. Resultado que parece mínimo, aun cuando la institución implementó desde hace 13 años su primer sistema de gestión (ISO 9001) y fue certificada por primera vez desde hace nueve.

Se consolida una propuesta que permite alinear los Sistemas de Gestión de la Calidad, Ambiental y de Seguridad y Salud en el trabajo implementados en el ICBF Regional Bogotá con las versiones ISO 9001:2015 e ISO 14001:2015, mediante actividades básicas y propias tanto de la institución como del diario mantenimiento de los sistemas.
La propuesta no requiere de mayores recursos, ya que los medios de consecución de cumplimiento se desarrollan a través de fórmulas básicas como son las actividades de sensibilización, uso de herramientas ya existentes (aplicativo ISOLUCIÓN), inserción de requisitos en los procedimientos ya implementados, control en la aplicación de actividades y controles ya establecidos; y alineación de planes existentes con necesidades estipuladas (plan institucional de capacitación); todo esto dado el sistema de gestión ya implementado.

Según lo anteriormente expuesto, formular una propuesta para alinear y mejorar el Sistema Integrado de Gestión del ICBF Regional Bogotá con las normas ISO 9001 y 14001 versiones 2015 y OHSAS 18001:2007, se considera una meta cumplible y alcanzable para la institución.

La implementación de la propuesta generaría mayor sinergia de los recursos disponibles, evitando reprocesos, aumentando la eficacia del Sistema Integrado de Gestión, aumentando el desempeño de la organización, y ante todo, aumentaría la satisfacción del cliente, aumentaría el control sobre los aspectos ambientales y disminuiría la posibilidad de incidentes y accidentes laborales.

Según la información recolectada, sería consecuente realizar investigaciones puntuales en cuanto a metodologías y herramientas para fortalecer la implementación, mantenimiento y mejora de los requisitos alineados con las relaciones con las partes interesadas y sensibilización de funcionarios.

Se consideran pertinentes estas dos temáticas según la percepción existente y debilidad operativa para fortalecer y controlar el cumplimiento de estos puntos dentro de un sistema integrado de gestión o al menos, dentro de un sistema de gestión único; aunado a la naturaleza pública de la institución en estudio, ya que las partes interesadas generan necesidades que están 
más allá de un mero vínculo comercial, siendo estas necesidades de carácter social y, por qué no decirlo, de derecho fundamental.

En cuanto a la sensibilización y concientización de los funcionarios ya sea en su aporte e influencia en el cumplimiento de requisitos al cliente, o de impacto con el medio ambiente o de autocuidado, evitando incidentes 0 accidentes que malogren su salud (en cualquiera de sus dimensiones), se deben trabajar una serie de variables de tipo psicológico que requieren un perfil específico para llegar a lograr resultados altamente calificados.

Es potestad de la institución extrapolar los resultados obtenidos por esta investigación, tanto a la sede de la Dirección General como a las 32 regionales restantes, con el objetivo de alinear las versiones 2015 tanto de la ISO 9001 como de la ISO 14001 con los requisitos establecidos en el Sistema Integrado de Gestión del ICBF.

\section{AGRADECIMIENTOS}

Agradezco la paciencia y las directrices de la ingeniera Sandra Giraldo; al ingeniero Guillermo Peña, le reconozco la convicción para llevar los trabajos de investigación a feliz término; al convenio USTAICONTEC, una alianza realmente estratégica; a Diana Patricia Arboleda Ramírez, directora del ICBF Regional Bogotá, permitiendo y apoyando la elaboración de esta investigación; a la ingeniera Sandra García Martínez y al ingeniero Fernando Velázquez Ávila, por su colaboración y apoyo; a mis compañeras Adriana Prieto y Gladys Sandoval; y a todos aquellos que de una u otra forma y tal vez a veces sin saberlo, aportaban conocimiento para obtener este resultado final.

\section{REFERENCIAS}

Hernández Sampieri, R., Fernández Collado, C., y Baptista Lucio, P. (2014). Metodología de la investigación. Sexta Edición. México D. F., México: McGraw-Hill.

ICBF. (2015). Resolución 10232 del 30 de noviembre de 2015. Instituto Colombiano de Bienestar Familiar. Bogotá D. C., Colombia: ICBF.

ICBF. (2016). Manual del Sistema Integrado de Gestión. Versión 3. Instituto Colombiano de Bienestar Familiar. Bogotá D. C., Colombia: ICBF.

Karapetrovic, S. (2003). Musings on integrated management systems. Measuring Business Excellence, 7(1), 4-13.

Karapetrovic, S., Casadesus, M., y Heras, I. (2010). Empirical analysis of integration within the standards-based integrated management systems. International Journal for Quality research, 4(1), 25-35.

Kauppila, O., Härkönen, J., y Väyrynen, S. (2015). Integrated HSEQ management systems: developments and trends. International Journal for Quality Research, 9(2), 231-242.

Portafolio. (15 de mayo de 2013). Solicitudes de certificaciones de calidad se duplican. Recuperado de: http://www.portafolio.co/negocios/empresas/ solicitudes-certificaciones-calidad-duplica-81454 >

Prieto Duarte, Y. A. (2015). Quality Forum, Red Internacional de Expertos en Calidad y Excelencia. Aspectos relevantes de la nueva ISO 14001 versión 2015. (48 diapositivas). Bogotá D. C., Colombia.

Tejada Losada, F., y Peña Guarín, G. (2009). Reflexiones sobre las características constitutivas de la gestión integral. SIGNOS, 1(2), 79-93. 
Melco Javier Leuro Rodríguez

Vera Jaimes, R. A. (2016). Quality Forum, Red Internacional de Expertos en Calidad y Excelencia. Jornada actualización de estándares. (36 diapositivas). Bogotá D. C., Colombia.

Zeng, S. X., Shi, J. J., y Lou, G. X. (2007). A synergetic model for implementing an integrated management system: an empirical study in China. Journal of cleaner production, 15(18), 1760-1767. 